\title{
Gastrointestinale Fehlbildungen und Erkrankungen des Neugeborenen - Teil 1
}

Ralf-Bodo Tröbs

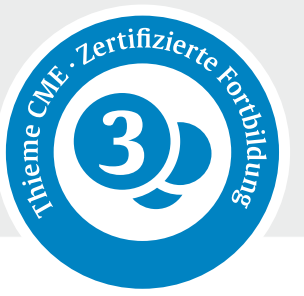

\begin{abstract}
Der Artikel gibt einen Überblick über kinderchirurgische Aspekte angeborener und erworbener Erkrankungen des Magen-Darm-Trakts. Für den Kliniker sind die pränatale Verdachtsdiagnose sowie Kenntnisse der postnatalen Leitsymptome essenziell. In diesen Fortbildungsbeitrag flossen zusammenfassende Darstellungen der letzten Jahre ebenso wie klinisch besonders relevante Sachverhalte ein [1-4].
\end{abstract}

\section{Vor der Geburt}

Die Einteilung angeborener und erworbener Erkrankungen des Magen-Darm-Trakts kann unter pathogenetischen und morphologischen Gesichtspunkten erfolgen ( $\triangleright$ Tab. 1).

Der pränatale Ultraschall erlaubt die Erkennung von ca. $60 \%$ der Fehlbildungen des Gastrointestinaltrakts (GIT). Atresien (griechisch atrelos, ohne Öffnung oder Lumen) sind durch einen angeborenen Verschluss oder eine hochgradige Einengung des Darmlumens gekennzeichnet. Die Entscheidungsfindung und fundierte Information der Eltern sollte interdisziplinär erfolgen. Berücksichtigt werden ethische Aspekte und die Risiken einer intrauterinen Gefährdung, die gegenüber einer zu frühen Geburt zu gewichten sind.

Gastrointestinale Fehlbildungen sind per se mit der Austragung eines Kindes vereinbar. Pränatale Eingriffe zur Behandlung von gastrointestinalen Fehlbildungen sind nicht etabliert. Eltern befürchten häufig eine spätere Einschränkung der Lernleistung sowie des Konzentrations- und Gedächtnisvermögens als Folge von Narkose und Operation. Hier sollte das Gespräch mit dem Kinderanästhesisten gesucht werden.

\footnotetext{
Merke

Derzeit ist die Datenlage nicht ausreichend, um eine Schädigung des frühkindlichen Gehirns durch Stress und Anästhetika auszuschließen.

Regionalanästhesiologische Verfahren stehen weniger in der Kritik (Spinal- oder Kaudalanästhesie). Diese haben sich in den letzten Jahren bei der Leistenherniotomie durchgesetzt $[5,6]$.
}

\section{Pathophysiologische Grundlagen}

Das Neugeborene beginnt bereits vor der Geburt zu schlucken. Röntgenologisch findet man nach 3 Stunden eine Luftfüllung des gesamten Dünndarms und nach 8-9 Stunden des Colon sigmoideum. Zur Röntgenbeurteilung der Darmluftverteilung nach der Geburt wird eine Latenzzeit von einigen Stunden abgewartet. Reife Neugeborene setzen innerhalb von 24 Stunden nach Geburt erstmalig Mekonium ab.

\footnotetext{
Merke

Ein Ausbleiben des Mekoniums über 24-48 Stunden nach der ersten Muttermilchmahlzeit ist ein Hinweis auf eine Darmpassagestörung.
}

Frühgeborene und insbesondere entwicklungsverzögerte Kinder (small for gestational age) tragen bei Vorliegen einer Mekoniumretention ein hohes Risiko für gastrointestinale Komplikationen. Eine rechtzeitige Er-

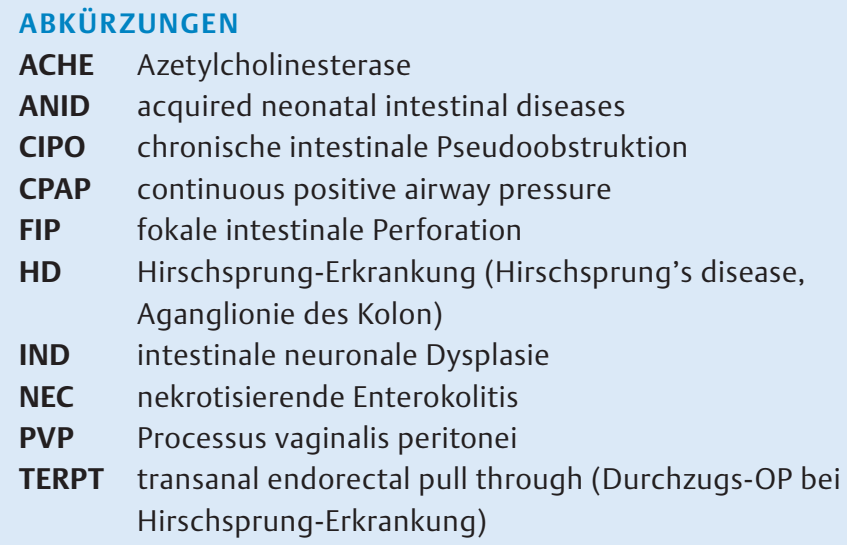


Tab. 1 Einteilung gastrointestinaler Fehlbildungen.

\begin{tabular}{|c|c|}
\hline Einteilung & Fehlbildung \\
\hline $\begin{array}{l}\text { embryologisch be- } \\
\text { dingte Fehlbildungen }\end{array}$ & $\begin{array}{l}\text { - tracheoösophageale Fistel/Ösophagusatresie } \\
\text { - Pylorusatresie } \\
\text { - Duodenalatresie bzw. -stenose } \\
\text { - Pancreas annulare } \\
\text { - Darmrotationsstörung } \\
\text { - Duplikatur, Mesenterialzyste } \\
\text { - anorektale Fehlbildung }\end{array}$ \\
\hline $\begin{array}{l}\text { Folgen fetaler } \\
\text { Störungen }\end{array}$ & $\begin{array}{l}\text { - Jejunum- und Ileumatresie } \\
\text { - Kolonatresie } \\
\text { - Mekoniumperitonitis }\end{array}$ \\
\hline $\begin{array}{l}\text { funktionell bedingte } \\
\text { Darmtransport- } \\
\text { störungen }\end{array}$ & $\begin{array}{l}\text { - Mekoniumobstruktion } \\
\text { - Mekoniumileus bei zystischer Fibrose }\end{array}$ \\
\hline $\begin{array}{l}\text { Kombination struk- } \\
\text { tureller und funktio- } \\
\text { neller Faktoren }\end{array}$ & $\begin{array}{l}\text { - Leistenhernie } \\
\text { - hypertrophe Pylorusstenose } \\
\text { - Volvulus bei Darmfixationsstörung }\end{array}$ \\
\hline Neuro-/Myopathien & $\begin{array}{l}\text { - Aganglionie (Hirschsprung) } \\
\text { - neuronale intestinale Dysplasie } \\
\text { - Megazystis, Mikrokolon, intestinales } \\
\text { Hypoperistaltik-Syndrom }\end{array}$ \\
\hline
\end{tabular}

kennung eines verzögerten Mekoniumabgangs eröffnet die Möglichkeit einer frühzeitigen Behandlung. Opioide können die Mekoniumausscheidung iatrogen verzögern [7].

Aufgepfropfte Zeichen einer gestörten Mekoniumpassage sind:

- gallige Magenreste

- Zunahme des Bauchumfangs

- Spannung und Schmerzhaftigkeit der Bauchdecke

- Darmsteifungen

- Rötung der Bauchwand

Cave

Im Falle therapeutischer Rektaleinläufe sind beim Neugeborenen phosphathaltige Klysmata streng zu meiden, da eine lebensbedrohliche Hyperphosphatämie, Hypokalzämie sowie metabolische Azidose drohen [8].

Bei akutem Abdomen bzw. lleus besteht ein intravasales Flüssigkeitsdefizit, bedingt durch Erbrechen, Durchfälle sowie eine Third-Space-Problematik. Bei ausgeprägter Hypovolämie sind Bolusgaben einer isotonen glukosefreien Infusionslösung effektiv. Insbesondere ischämiebedingte Schmerzen sind schwerwiegend und sollten unter Einsatz von Opioiden eingedämmt werden.
Zu den Standardmaßnahmen gehören weiterhin:

- Einlage einer ausreichend fördernden naso-gastrischen Sonde in Form einer Heberdrainage, ggf. Aspiration des Mageninhalts

- fakultativ ein transurethraler Katheter

- Bei Vorliegen einer systemischen Entzündungsreaktion (systemic inflammatory response syndrome, SIRS), V.a. Durchwanderung oder Perforation der Darmwand ist eine kalkulierte Antibiotikatherapie (Spektrum gramnegativ, -positiv sowie anaerob) erforderlich.

\section{Merke}

Die Magensonde dient der Entlastung des oberen GIT. Sie stellt jedoch keinen Aspirationsschutz dar.

Grundsätzlich sind Darmresektionen so sparsam als möglich auszuführen. Ziel ist die Bewahrung der enteralen Autonomie, d.h. Erhalt des Vermögens einer oralen Ernährung ohne zusätzliche parenterale Flüssigkeits- bzw. Elektrolyt- und Nährstoffsubstitution. Intraoperativ wird die Länge des verbleibenden Dünndarms, beginnend an der Flexura duodenojejunalis (TreitzBand), gemessen (Fadenmethode).

\section{Merke}

Die Ileozökal-Klappe (Bauhin) sollte möglichst erhalten bleiben.

Unter funktionellem Gesichtspunkt resultiert ein Kurzdarm, wenn der erhaltene Darm nicht ausreichend Nährstoffe, Wasser und Elektrolyte zu absorbieren vermag. Hochkritische Darmlängen liegen unter $40 \mathrm{~cm}$ Rest-Dünndarm. Betroffene benötigen nach überstandenem Akutereignis zunächst eine längerfristige parenterale Ernährung - jedoch kann ein relevanter Anteil von der parenteralen Ernährung entwöhnt werden. Die funktionelle Anpassung (enterale Adaptation) des verbleibenden Darmes benötigt einen Zeitraum bis zu 4 Jahren [9].

Ursachen für einen Kurzdarm sind insbesondere:

- Volvulus

- Dünndarmatresien

- nekrotisierende Enterokolitis (NEC)

- chronische intestinale Pseudoobstruktion (CIPO)

Besondere Probleme ergeben sich aus der pathogenen mikrobiologischen Besiedlung des verbleibenden Darmes. Limitierend für das Körperwachstum und die Entwicklung sowie quoad vitam sind insbesondere die kurzdarmassoziierte chronische Lebererkrankung sowie enterogene und katheterassoziierte Septitiden. 


\section{Pylorusatresie, Magenmembran}

Der Verschluss des Magenausgangs tritt sporadisch oder in Verbindung mit einer Epidermolysis bullosa auf. Das Röntgenbild zeigt eine überblähte Magenblase mit Flüssigkeitsspiegel. Distal davon fehlt die Luft (Ausnahme inkomplette Magenmembran). Gefahren leiten sich aus einer drohenden Magenperforation ab. Die Behandlung kann in einer Pylorusresektion und End-zuEnd-Anastomose oder Seit-zu-Seit-Gastroduodenostomie bestehen.

\section{Duodenalatresie bzw. -stenose, Pankreas annulare}

Die Häufigkeit beträgt 1,5-2 auf 10000 Geburten. Embryologisch handelt es sich um die Folge einer gestörten epithelial-mesenchymalen Interaktion bei der Rekanalisierung des ursprünglich epithelial verschlossenen Duodenums (Tandler-Theorie). Pränatal finden sich ein Polyhydramnion und eine Aufweitung von Magen und Duodenum.

Merke

An die erhöhte Prävalenz eines Vitium cordis sowie einer Trisomie 21 ist zu denken.

Postnatal sind galliges Erbrechen und eine Vorwölbung des Oberbauchs Leitsymptome. Besonders seltene Atresien oralwärts der Vater-Papille sind mit klarem Rückfluss verbunden. Die Nativ-Röntgenaufnahme des Magens offenbart die doppelte Spiegelbildung von Magen und Duodenum (double bubble). Zur besseren Sichtbarmachung können vor dem Röntgen einige Milliliter Luft über die naso-gastrische Sonde instilliert werden. In unklaren Fällen bringt ein wasserlösliches Kontrastmittel zusätzlichen diagnostischen Gewinn.

Morphologische Formenvarianten der Duodenalatresie sind Membranen (mit oder ohne Öffnung) oder die Lumen- bzw. Kontinuitätsunterbrechung ( $\mathbf{A b b} \mathbf{1}$ ). Standardmethode der chirurgischen Therapie ist die Duodeno-Duodenostomie in Form der Diamantanastomose. Sie kann offen oder bei spezieller Expertise laparoskopisch durchgeführt werden. Eine transnasal eingeführte transanastomotische Sonde erlaubt eine frühzeitige postoperative enterale Ernährung. Alternativ ist eine Membranexzision möglich (per Enterotomie oder bei größeren Kindern auch endoskopisch endoluminal).

Cave

Bei der Membranexzision ist auf die Unverletztheit der Papille strikt zu achten!

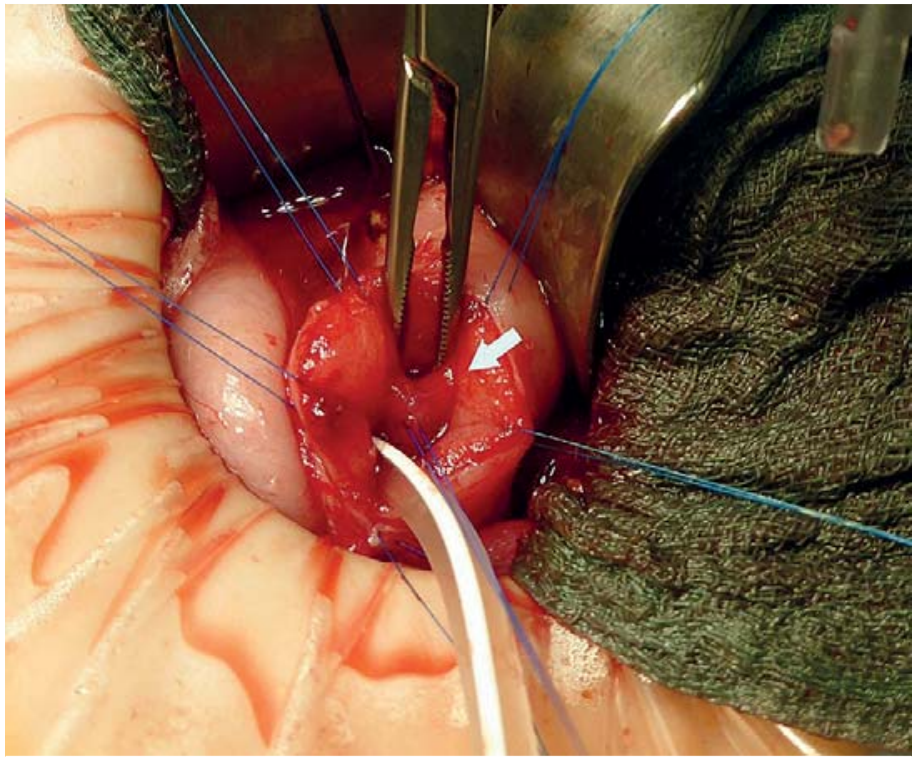

Abb. 1 Duodenalmembran. Eröffnetes Darmlumen: fleischige Membran vor den Branchen der Pinzette. Die Magensonde kommt von oben und wird transanastomotisch platziert.

Eine Verschmälerung (tapering) eines Megaduodenums ist in seltenen Fällen erforderlich. Eine präduodenale Pfortader (persistierende linke Vitellin-Vene) kommt bei Heterotaxie bzw. Situs-Asymmetrie vor. Sie verläuft vor dem Pankreas, und sie ist meist von einer intrinsischen Obstruktion begleitet.

Das Pankreas annulare als Ursache einer Duodenalstenose resultiert aus einer fehlerhaften Verschmelzung beider embryonaler Pankreasananlagen. Das Pankreas umschließt das Duodenum ringförmig ( $\mathbf{A b b}$. 2). Die Behandlung besteht in einer Überbrückung in Form der Duodeno-Duodenostomie.

Bei der Ladd-Assoziation bewirken embryonale peritoneale Bänder, ausgehend von einem hochstehenden Zökum (Coecum altum), eine äußere Duodenalkompression. Die zusätzlich schmalbasige Aufhängung des Mitteldarms prädestiniert zum Volvulus. Die Operation umfasst neben der Durchtrennung der Ladd-Bänder die Mobilisation der mesenterialen Verwachsungen und Positionierung des Darmes in Non-Rotation. In 2-7\% der Fälle ist ein Volvulus-Rezidiv möglich. Auch eine Nahtfixierung des derotierten Darmes bietet keinen sicheren Schutz dagegen.

Merke

Die Ladd-Prozedur gibt den Patienten eine größtmögliche Sicherheit gegen ein Volvulusrezidiv, jedoch bedarf es einer Information über mögliche Symptome und Dringlichkeit bei V.a. Re-Volvulierung. 


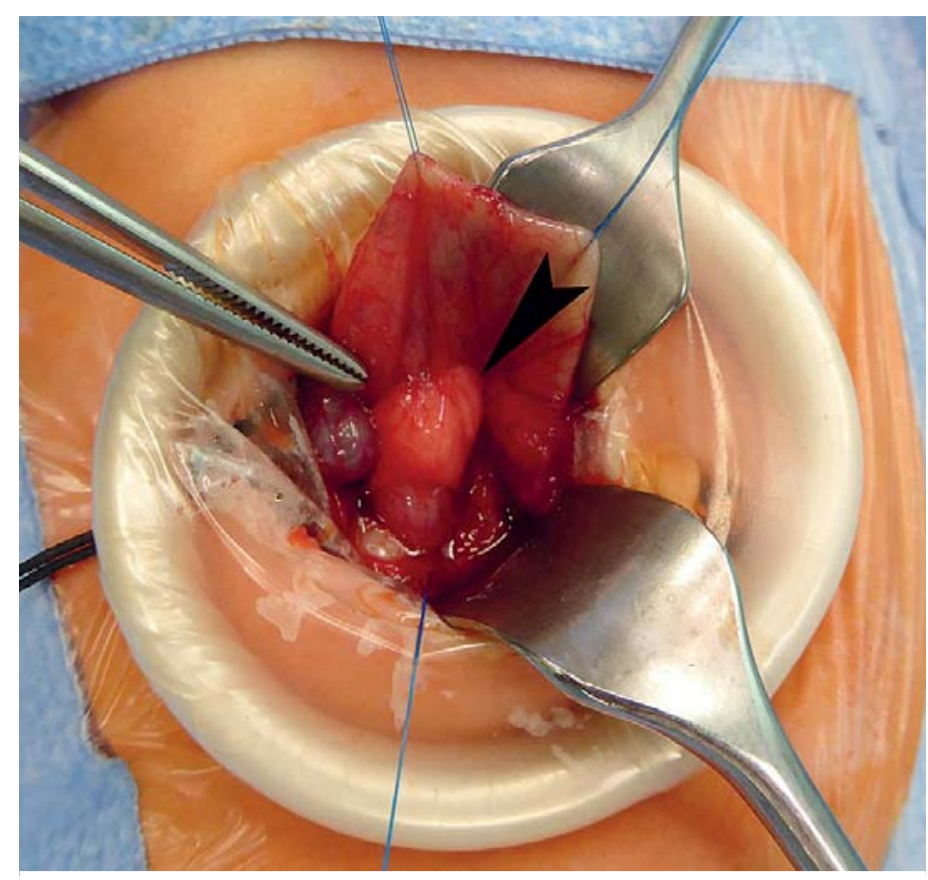

- Abb. 2 Pancreas annulare. Der Magen ist mit Haltefäden aufgespannt. Das Pankreas umschließt ringförmig das Duodenum. Bypass-Anastomose mittels Duodeno-Duodenostomie.

\section{FALLBEISPIEL}

Pränatal bestand ein ausgeprägtes Polyhydramnion bei V.a. Double Bubble im Ultraschall. Wegen mütterlicher Probleme erfolgt die Sectio-Entbindung in der 29. Schwangerschaftswoche mit einem Geburtsgewicht von $800 \mathrm{~g}$. Diese Diagnose bestätigt sich auch im Nativ-Röntgen des Abdomens. Nach der Geburt bestätigt sich eine Trisomie 21, und das Herzecho ergibt einen perimembranösen Ventrikelseptumdefekt. Über die Magensonde ist grünes Sekret aspirierbar.

Das Frühgeborene ist beatmet, so dass am 4. Lebenstag die Off-Site-Operation im kooperierenden Perinatalzentrum erfolgt. Es wird eine Oberbauch-Laparotomie rechts vorgenommen. Eine Durchtrennung des noch offenen Lig. teres hepatis ist nicht erforderlich. Intraoperativ bestätigt sich eine Duodenalatresie ohne Verbindung zwischen dem dilatierten oberen Segment und dem „abgekoppelten“ nachgeschalteten, schmalkalibrigen Duodenum. Eine Rotationsanomalie oder sonstige weitere Auffälligkeiten finden sich nicht. Aufgrund der lokalen Verhältnisse wird eine Seit-zu-Seit-Anastomose mit Vicryl $7 \times 0$ unter lupenchirurgischer Vergrößerung ausgeführt. Nach Fertigstellung der Hinterwand schieben wir eine $4 \mathrm{CH}$ Ernährungssonde über die Anastomose.

Der weitere Verlauf ist befriedigend. Das Kind bleibt an der Beatmung. Nach 4 Tagen erfolgt die Gabe von $5 \%$-Glukoselösung über die Sonde. Der weitere Nahrungsaufbau geschieht mit Muttermilch. Eine Spontanpassage über die Anastomose stellt sich ein.

\section{Dünn- und Dickdarmatresie}

Auch hier sind es sekundäre Atresien nach fetalen Gefäßverschlüssen (Embolie, Strangulation, Segmentverdrehung) des Darmes. Besondere Kausalitäten finden sich bei Kindern mit schweren Herzfehlern oder nach Zwillingstransfusionssyndrom. Bereits intrauterin kann die Darmdilatation sonografisch gesehen werden.

Leitsymptome sind die stetig zunehmende Vorwölbung des Bauches und galliges Erbrechen. Die Nativ-Röntgenuntersuchung zeigt meist mehrere Spiegel und ein luftleeres unteres Abdomen. Bei Jejunumatresie ist das gefüllte, wandverdickte und stark erweiterte obere blinde Dünndarmsegment häufig als wurstförmige Resistenz tastbar ( $\triangleright$ Abb. $\mathbf{3}$ ).

Morphologisch unterscheidet man membranöse, strangförmige oder komplette Atresieformen (nach Veccia, Grosfeld und West):

- Typ I: intraluminale Membran

- Typ II: Strang zwischen beiden atretischen Enden unter Erhaltung des Mesenteriums

- Typ IIla: atretische Enden sind bei Mesenterialdefekt nicht miteinander verbunden

- Typ IIlb: Christmas Tree oder Apple Peel

- Typ IV: multiple Atresieformen nebeneinander vorliegend ( $\triangleright$ Abb.4)

Atresien vom Typ III (aboraler Darm apfelschalenartig um das Zentralgefäß gewunden) werden pathogenetisch auf einen intrauterinen Volvulus oder Gefäßverschluss im Versorgungsgebiet der distalen A. mesenterica superior zurückgeführt. Der mittlere Dünndarm ist untergegangen, a priori besteht eine Kurzdarmsituation. Die Blutversorgung des verbliebenen terminalen lleum erfolgt über einen Ast der ileokolischen Arterie.

Die bevorzugte Behandlungsoption bei Dünndarmatresien besteht in der primären Wiederherstellung der Darmkontinuität mittels End-to-Back-Anastomose. Es ist fast immer erforderlich, das megalisierte präatretische Segment sparsam zu resezieren. Ein Tapering erlaubt zusätzlich die Verschmälerung des präatretischen Darmes. Weiterhin kann der postatretische Darm antimesenterial spatuliert, d.h. längs eingeschnitten werden. Eine transnasal eingelegte transanastomotische Sonde oder eine temporäre Katheterjejunostomie nach dem Witzel-Prinzip erlauben die frühzeitige enterale Ernährung. Allerdings gibt es Untersuchungen, die die Effizienz dieses Vorgehens anzweifeln.

Kolonatresien sind besonders selten. Eine Auftreibung des Bauches und gallig-mekoniumhaltiges Erbrechen sind die Leitsymptome. Die Behandlungsprinzipien entsprechen dem bereits beschriebenen Vorgehen, ggf. wird ein temporäres Stoma angelegt. 


\section{Merke}

Wann immer möglich, sollte der ileokolische Übergang mit der Bauhin-Klappe erhalten werden.

Nach der Operation einer hohen Atresie bewährt sich eine Jejunalsonde zwecks frühzeitigen enteralen Nahrungsaufbaus. Eine orale Nahrungszufuhr erfolgt in Absprache mit dem Kinderchirurgen nach einigen Tagen, meist nach Absetzen der ersten Portionen frischen Stuhls.

\section{Duplikaturen}

Duplikaturen kommen entlang des gesamten Darmrohrs vor. Es handelt sich um sphärische oder zylindrische Doppelungen des Lumens, deren Wand Schleimhaut und Muskulatur enthält. Duplikaturen sind meist vom Darmlumen getrennt oder können mit dem Lumen in offener Verbindung stehen.

Die Embryologie ist nicht schlüssig zu erklären, so dass eine Reihe embryologischer Theorien ins Feld geführt werden:

- Split-Notochord-Theorie

- Persistenz embryonaler Divertikel

- Störung der Rekanalisierung des Darmes

Die Diagnose erfolgt häufig pränatal durch Ultraschall. Am häufigsten betroffen sind Ileum und Jejunum, gefolgt von Ösophagus sowie Kolon.

Nach der Geburt bleiben Duplikaturen zunächst asymptomatisch. Durch Größenzunahme, Sekundärinfektion sowie infolge eingesprengter dystoper Magenbzw. Pankreasschleimhaut entstehen Komplikationen, z.B: Darmpassagestörung, Ruptur und Peritonitis. Klinisch tastet man nicht selten eine gut verschiebliche Raumforderung im Bauch. Meist genügt ein Ultraschall zur weiteren Spezifizierung.

Eine elektive Operationsindikation besteht aufgrund der zu befürchtenden Komplikationen. Methode der Wahl ist die kontinuitätserhaltende Resektion, jedoch lässt sich eine Resektion des betroffen Darmabschnitts nicht immer vermeiden. Differenzialdiagnosen sind insbesondere Mesenterialzysten, Lymphangiome sowie Ovarialzysten.

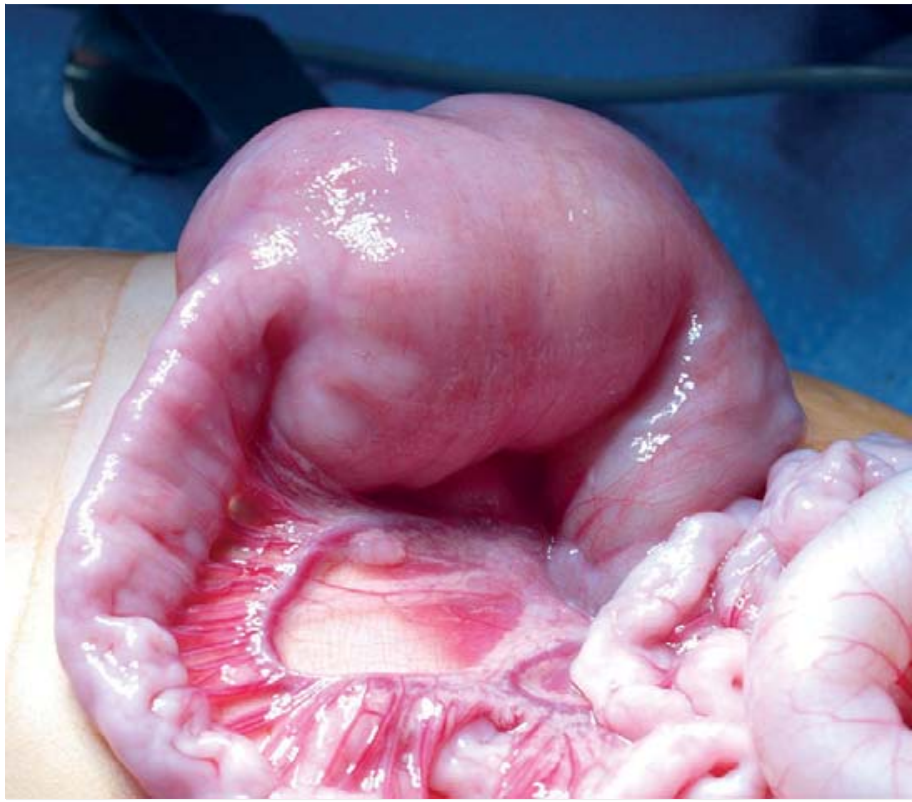

- Abb. 3 Jejunalatresie. Infolge einer Membran ausgeprägte prästenotische Dilatation und Wandhypertrophie des Jejunum. Quelle: Nissen M, Dettmer P, Thränhardt R et al. Congenital jejunal membrane causing transient pseudohypoaldosteronism and hypoproteinemia in a 7-weekold infant. Klin Padiatr 2017; 229: 302 - 303. doi:10.1055/s-0043113570.

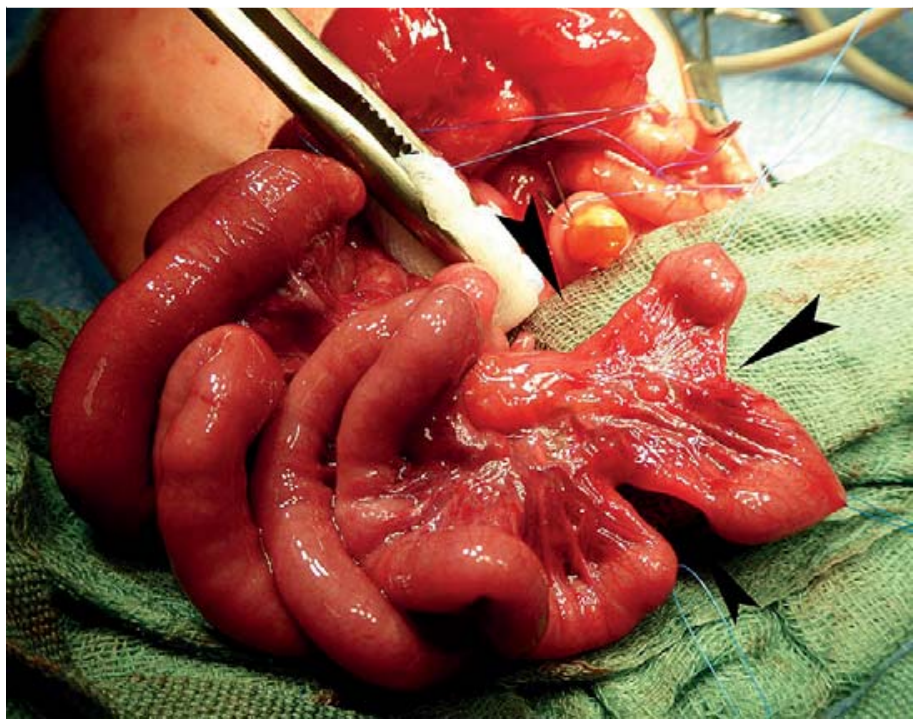

- Abb.4 Typ IV einer Jejunalatresie. Die Kontinuität des Jejunums ist mehrfach komplett unterbrochen. Verkürzung der Gesamtlänge des Dünndarms. 


\section{Darmrotations- und Fixations- störungen, Volvulus}

Ursächlich sind Störungen im Rahmen der Hernierung des embryonalen Mitteldarms in das extraembryonale Zölom und der Rückverlagerung in die Bauchhöhle (Nabelschleifenstörung). Damit verbunden ist die 3-dimensionale und gegenläufige embryonale Darmdrehung, die zu einer Anhaftung des duodeno-jejunalen Übergangs im linken Oberbauch sowie einer Lage des ileo-zökalen Übergangs im rechten Unterbauch führt. Wird dieser Prozess gestört, so resultieren eine Mal- bzw. Non-Rotation, begleitet von einer mangelhaften Fixierung des Darmes: Mesenterium ileocolicum commune. Ein pendelartig am schmalen Mesenterialstiel aufgehängter Mitteldarm prädestiniert zur Volvulierung um die Drehachse der oberen Mesenterialgefäße.

Der Formenreichtum intestinaler Fixations- und Rotationsstörungen ist groß. So gibt es neben der ausbleibenden Rotation (Non-Rotation) Fälle mit inkompletter oder sehr selten auch inverser Rotation sowie innere Hernien (mesokolische paraduodenale Hernien).

Leitsymptom des Volvulus ist das meist gallige Erbrechen aus dem scheinbaren Wohlbefinden heraus.

\footnotetext{
Cave

Bei Mitteldarmvolvulus droht die subtotale hämorrhagische Darminfarzierung innerhalb weniger Stunden!
}

Bei Früh- und Neugeborenen ist die Schmerzsymptomatik meist gering. Dagegen zeigen größere Kinder starke Ischämieschmerzen, die zyklisch im Rahmen einer voranschreitenden Volvulierung eintreten können. In diesem Zusammenhang ist vor Diagnosen wie „zyklisches Erbrechen, Abdominalmigräne“ oder „psychogenen, hysterischen Anfällen“ zu warnen.

Beim Volvulus spielt der Zeitfaktor eine entscheidende Rolle und im Entscheidungsfalle ist der klinische Verdacht ausschlaggebend. Das Nativ-Röntgenbild ist in ca. $20 \%$ der Fälle mit Volvulus unauffällig!

Merke

Bei jeder Duodenalobstruktion des Neugeborenen und Säuglings ist ein Volvulus auszuschließen!
Mit dem Ultraschall inklusive farbkodiertem Doppler steht ein zügig anzuwendendes und sensitives Verfahren zur Verfügung. Prinzipiell liegt die Vena mesenterica superior rechtsseitig der Arteria mesenterica superior (bei Volvulus liegt die Vene linksseitig oder vor der Arterie). Das klassische Ultraschallzeichen des Mitteldarmvolvulus ist das Whirlpool-Zeichen, d.h. die Verdrehung der Gefäße des Mesenterialstiels im Uhrzeigersinn.

Bei oraler Kontrastmittelgabe weist eine Rechtslage der Flexura duodenojejunalis (im Normalfall linksseitig der Wirbelsäule) auf eine Rotationsstörung hin. Ein hoher Duodenalspiegel oder Kontrastmittelabbruch in Höhe Pars 3 duodeni sind Zeichen eines Volvulus. Bei chronischem Verlauf liefern Schnittbildverfahren mit Kontrastmitteldarstellung der Gefäße die Diagnose. Der retrograde Röntgen-Kolonkontrasteinlauf liefert als Zeichen des Volvulus ein hochstehendes Zökum und einen Kontrastmittelabbruch im terminalen lleum.

Bei Frühgeborenen ist die isolierte Verdrehung einer Darmschlinge in Form des segmentalen Volvulus typisch. Dies ist infolge entwicklungsbedingt laxer und enger Darmaufhängung möglich. Eine iatrogene Triggerung (z. B. durch CPAP-Atemunterstützung, Bauchmassage oder Beckenrotation) wird diskutiert. Ein galliger Rückfluss und das eingesunkene, röntgenologisch fast luftleere Abdomen sowie eine Laktazidose (nicht obligat!) sind hinweisend. Da die torquierte Darmschlinge häufig nekrotisch ist, stehen Resektion und Anastomose an. Der Darmverlust gestaltet sich geringer als beim klassischen Mitteldarmvolvulus.

Spätsymptome des Volvulus sind:

- Hämatin

- Hämochezie

- Paralyse des Darmes

Merke
Multiple Röntgenspiegel deuten auf eine fortge-
schrittene Infarzierung hin.

Bei V.a. Volvulus ist die umgehende Operation (ggf. Laparoskopie, meist offen) unabdingbar. Häufig sind laparoskopische Manipulationen (LADD-Manöver) aufgrund überdehnter und rigider Darmschlingen erschwert, so dass laparotomiert werden muss. Bei einem Strangulationsileus durch angeborene Briden (z. B. Reste der Vitellin-Arterien, Ductus omphaloentericus) ist die Laparoskopie zielführend. 
Bei Mitteldarmvolvulus und hämorrhagischer Infarzierung ist eine Second-Look-Operation nach 24-36 Stunden eine Option zur Erhaltung des Darmes (ggf. ist auch die Clip-and-drop-Technik anzuwenden).

Kontrovers wird die Entfernung der Appendix bei Rotationsanomalien des Darmes diskutiert. Aus infektimmunologischen Gründen ziehen wir es vor, die Appendix nicht zu entfernen, da diese einen Rückzugsort der normalen Darmflora bei Enteritis ist [10]. Allerdings sollten Eltern und ggf. auch das verständige Kind auf die Möglichkeit einer Links-Appendizitis hingewiesen werden.

\section{Hinweis}

Weitere Fehlbildungen und Erkrankungen werden in Teil 2 des Beitrags in der nächsten Ausgabe (2/2019) behandelt.

\section{KERNAUSSAGEN}

- Fehlbildungen des oberen Gastrointestinaltrakts werden überwiegend pränatal im Ultraschall diagnostiziert.

- Atresien und Darmfixationsstörungen sind am häufigsten anzutreffen.

- Galliges Erbrechen ist ein Alarmzeichen mit der Konsequenz einer weiterführenden Diagnostik sowie der Einlage einer nasogastrischen Sonde.

- Typisch für die Duodenalatresie ist die Double Bubble im Ultraschall und auf der Röntgenaufnahme; die Operation erfolgt mit aufgeschobener Dringlichkeit.

- Atresien des unteren Dünndarms und des Kolons manifestieren sich häufig verzögert durch Auftreibung des Darmes und galliges bzw. fäkulentes Erbrechen.

- Multiple Dünndarmspiegel bei fehlender Belüftung des unteren Mittel- und Enddarmes belegen postnatal eine tiefere Dünndarmatresie.

- Bei Dünndarmatresien besteht ein Trend zur primären Anastomose, wenn immer möglich.

- Duplikaturen sind initial oft symptomarm, erweisen sich jedoch später als komplikationsträchtig, so dass eine elektive Operationsindikation besteht.

- Darmrotaions- und Fixationsstörungen prädestinieren zum Mitteldarmvolvulus.

- Ein V.a. Volvulus erfordert die Abklärung innerhalb einer Stundenfrist. Bei langstreckiger hämorrhagischer Infarzierung ist nach Detorsion eine Second-Look-Laparotomie zu erwägen.
Schlüsselwörter

akutes Abdomen, Ileus, Neugeborenes, Atresie, Duplikatur

Interessenkonflikt

Der Autor gibt an, dass keine Interessenkonflikte vorliegen.

\section{Autorinnen/Autoren}

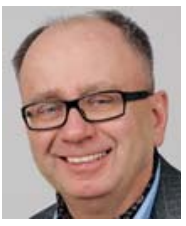

\section{Ralf-Bodo Tröbs}

Prof. Dr. med., Jahrgang 1956; Studium der Humanmedizin und Facharztweiterbildung in Leipzig, Assistenzart für Pathologie über 2 Jahre; 1995 Habilitation, Auslandserfahrungen in Laos, Jemen. Seit 2006 Direktor der Klinik für Kinderchirurgie in Herne, Witten, Ruhr-Universität Bochum. Zusatzweiterbildung: Intensivmedizin, Schwerpunkte Neugeborenen- und Abdominalchirurgie, Kinderurologie, perioperative Physiologie.

\section{Korrespondenzadresse}

Prof. Dr. med. Ralf-Bodo Tröbs

Marienhospital Witten, St. Elisabeth Gruppe

Ruhr-Universität Bochum

Klinik für Kinderchirurgie

Marienplatz 2

54452 Witten

E-Mail: ralf-bodo.troebs@elisabethgruppe.de

Wissenschaftlich verantwortlich gemäß Zertifizierungsbestimmungen

Wissenschaftlich verantwortlich gemäß Zertifizierungsbestimmungen für diesen Beitrag ist Prof. Dr. med. Ralf-Bodo Tröbs, Witten.

Literatur

[1] Hall N], Eaton S, Pierro A. The evidence base for neonatal surgery. Early Human Developm 2009; 85: 713 - 718

[2] Krause H, Rißmann A, Haß HJ et al. Was muss der (Viszeral-) Chirurg von der Kinderchirurgie wissen - kinderchirurgische Aspekte in der ( Viszeral-)Chirurgie. Zentralbl Chir 2011; 136: 458-547

[3] Wessel L, Fuchs J, Rolle U. Korrektur angeborener Fehlbildungen in der Kinderchirurgie. Dtsch Ärtzebl Int 2015; 112 : $357-364$

[4] Tam PKH, Chung PHY, Peter SD et al. Advances in paediatric gastroenterology. Lancet 2017; 390: 1072 - 1082

[5] Weber F, Vlot J, Wijnen R. Safeguarding infant brains. A multidisciplinary challenge - results of a survey, update on current scientific evidence, and recommendations on how to deal with possible anesthetic drug neurotoxicity. Eur ] Pediatr Surg 2018; 28: 400 - 405 
[6] Prato AP, Casaccia G, Arnoldi R. Timing and management of inguinal hernia in the premature baby. Eur J Pediatr Surg 2017; 27: 472-477

[7] Gupta AK, Guglani B. Imaging of congenital anomalies of the gastrointestinal tract. Indian J Pediatr 2005; 72: 403 414

[8] Bekkali N, Hamers SL, Schipperus MR et al. Duration of meconium passage in preterm and term infants. Arch Dis Child Fetal Neonatal Ed 2008; 93: F376-F379
[9] Marek I, Benz K, Kusnik S et al. Phosphatintoxikation nach Klysmagabe - eine lebensgefährliche iatrogene Komplikation. Klin Pädiatr 2015; 227: 235-238

[10] Capriati T, Giorgio D, Fusaro F et al. Pediatric short bowel syndrome: Predicting four-year outcome after massive neonatal resection. Eur J Pediatr Surg 2018; 28: 455-463

Bibliografie

DOI https://doi.org/10.1055/a-0821-8279

Neonatologie Scan 2019; 08: 59-69

(c) Georg Thieme Verlag KG Stuttgart · New York

ISSN 2194-5462 


\section{Punkte sammeln auf CME.thieme.de}

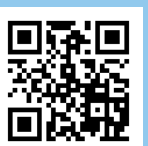

Diese Fortbildungseinheit ist in der Regel 12 Monate online für die Teilnahme verfügbar.

Den genauen Einsendeschluss finden Sie unter https://eref.thieme.de/CXCF5A7.

Sollten Sie Fragen zur Online-Teilnahme haben, finden Sie unter https://cme.thieme.de/hilfe

eine ausführliche Anleitung. Wir wünschen viel Erfolg beim Beantworten

der Fragen!

Unter https://eref.thieme.de/CXCF5A7 oder über den QR-Code kommen Sie direkt zur Startseite des Wissenstests.

VNR 2760512019156640276

\section{Frage 1}

Galliges Erbrechen und zunehmende Vorwölbung des Abdomens führen zur Anlage einer naso-gastrischen Sonde. Welche Aussage trifft zu?

A Es reicht, eine möglichst dünne und geschmeidige Sonde zu legen.

B Die Magensonde ist ein sicherer Aspirationsschutz.

C Magensonden zur Entlastung sind wirkungslos. Hier hilft sich die Natur mittels Erbrechen selbst!

D Es ist wichtig, die Sonde zwischenzeitlich abzustöpseln.

E Die Sonde soll ein ausreichendes Kaliber aufweisen und zur Gewährleistung der Drainage stets offen bleiben.

\section{Frage 2}

Aufgrund auffälliger intrauteriner Darmdilatation und Vermehrung der Echogenität der Darmwand soll das Abdomen des Neugeborenen geröntgt werden. Interessant sind besonders der untere Dünndarm sowie das Kolon. Welche Aussage trifft zu?

A Das Nativ-Röntgen kann gleich nach der Geburt erfolgen.

B Am günstigsten ist die Wangensteen-Aufnahme im „Hängen“.

C Es sollten möglichst einige Stunden vergangen sein, bis die Aufnahme durchgeführt wird.

D Eine Röntgenaufnahme ohne orales Kontrastmittel erübrigt sich, da nicht aussagekräftig.

E Zur Beurteilung der Darmluftverteilung sollte gleich im seitlichen Strahlengang untersucht werden. Eine a.p.-Aufnahme ist nicht von Nöten.

\section{Frage 3}

Ein Neugeborenes zeigt bereits unter der Geburtsentwicklung flächige Hautablösungen. Zudem fiel ein Polyhydramnion auf. An welche Konstellation denken Sie?

A Eine gramnegative Infektion ist sehr wahrscheinlich.

B Das Polyhydramnion wird durch eine intrauterine Infektion begünstigt.

C Die Hautablösungen ergeben sich als Folge heftiger intrauteriner Kindsbewegungen.

D Es ergeben sich Hinweise auf eine Epidermolysis bullosa und eine Pylorusatresie.

E Das Polyhydramnion wurde sehr wahrscheinlich durch eine Ösophagusatresie hervorgerufen.

\section{Frage 4}

Nach pränatalem V.a. Duodenalatresie wird ein Neugeborenes mit einem Körpergewicht von $2600 \mathrm{~g}$ vaginal entbunden. Pränatale Phase und Geburt verliefen komplikationslos. Welche Begleitfehlbildungen sind insbesondere zu berücksichtigen?

A VACTERL-Assoziation

B Double Bubble sowie Oligohydramnion

C Trisomie 18 und Nierenfehlbildungen

D Trisomie 21 und Herzfehlbildungen

E Duodenalatresien treten meist isoliert auf; verstärkte diagnostische Anstrengungen nach reibungsloser Geburt sind nicht nötig. 


\section{Punkte sammeln auf CME.thieme.de}

Fortsetzung ...

\section{Frage 5}

Für Neugeborene werden phosphatfreie Klysmen angewendet. Welche Begründung hierfür ist richtig?

A Infolge der Hyperosmolarität droht bei Phosphatklysmen eine Darmruptur.

B Phosphathaltige Klysmen sind wenig wirksam, und durch den Phosphateinstrom entsteht eine schwere Begleitalkalose.

C Infolge des Phosphats kommt es zu Steinbildungen in den Harnwegen.

D Es handelt sich um eine geringe Phosphatdosis, die i.d.R. renal kompensiert wird.

E Es droht eine lebensbedrohliche Phosphatintoxikation mit Auswirkungen auf das Blutkalzium und Gefährdung der Nierenfunktion.

\section{Frage 6}

Bei der Operation eines Neugeborenen mit Dünndarmatresie findet sich ein Typ III b. Eine Anastomose wird erzielt. Welche Konstellation bezüglich der Darmlänge und -funktion ist zu erwarten?

A Wie auch bei Typ I oder II kann man eine normale Entwicklung erwarten.

B Aus embryologischen Gründen fehlt Darmlänge in relevanter Größenordnung.

C Zur Beurteilung der perspektivisch zu erzielenden Darmleistung bedarf es der Darmadaptation über mindestens 2-3 Wochen.

D In diesen Fällen fehlt die lleozökalklappe, was die Situation verschlimmert.

E Eine Assoziation mit einer Aganglionie ist typisch, was die Darmlänge zusätzlich potenziell beeinflusst.

\section{Frage 7}

Im Pränatalultraschall fand man bei einem Mädchen eine zystische Raumforderung im Bauch. Nach der Geburt tastet man eine gut verschiebliche Raumforderung im rechten Unter- bis Mittelbauch. Symptome bestehen nicht. Welche Aussage trifft am besten zu?

A Es handelt sich am ehesten um eine Hydronephrose.

B In diesen Fällen besteht fast immer eine Ovarialzyste und es kann abgewartet werden.

C Unmittelbar nach Geburt ist die operative Freilegung unumgänglich.

D Mit Ultraschall kann sowohl die Organzuordnung getroffen als auch die Durchblutung verifiziert werden. Im Falle einer ileozökalen Duplikatur ergeben sich keine weiteren Maßnahmen.

E Wenn der Ultraschall eine intestinale Duplikatur zeigt, sollte eine chirurgische Behandlung vor Eintreten von Komplikationen eingeleitet werden.

\section{Frage 8}

Ein 8-jähriges Kind wird mit Erbrechen, Unterbauchschmerzen, Temperaturerhöhung im leicht reduzierten Zustand vorgestellt. Bei der Untersuchung fällt eine Laparotomienarbe auf. Der Druckschmerz ist umschrieben links unten zu lokalisieren. Die Eltern bestätigen, dass im Säuglingsalter eine Darmverdrehung bestanden habe, die mittels Operation beseitigt wurde. Auf Nachfrage berichten die Eltern weiterhin, dass der Wurmfortsatz nicht entfernt worden war. Welche diagnostischen Erwägungen drängen sich auf?

A An eine „Linksappendizitis“ infolge Non-Rotation muss ebenso wie an eine Re-Volvulierung gedacht werden.

B Die Befunde sprechen ganz klar für eine Gastroenteritis.

C Gerade die nicht sehr ausgeprägte Minderung des Allgemeinzustands spricht für eine Re-Volvulierung.

D Bei galligem Erbrechen sind Sonografie und ggf. Röntgenuntersuchung nicht erforderlich.

E Eine Kontrolluntersuchung am Folgetag ist ausreichend, um den Verlauf zu beurteilen. 


\section{Punkte sammeln auf CME.thieme.de}

\section{Fortsetzung ...}

\section{Frage 9}

Nach einem Mitteldarmvolvulus im Alter von 2 Monaten konnten $45 \mathrm{~cm}$ Dünndarm sowie der ileozökale Übergang erhalten werden. Postoperativ ist eine teilparenterale Ernährung erforderlich. Der orale Nahrungsaufbau ist nur schrittweise zu erzielen. Welche Situationseinschätzung ist angebracht?

A $43 \mathrm{~cm}$ Dünndarm reichen in jedem Fall für eine intestinale Autonomie.

B Darmlängen unter $70 \mathrm{~cm}$ haben eine schwierige Prognose, so dass die Eltern über eine lebenslange parenterale Ernährung informiert werden müssen.

C Die absolute Darmlänge ist allein nicht ausschlaggebend. Im vorliegenden Fall ist es günstig, dass die Bauhin-Klappe erhalten werden konnte.

D Aufgrund der Vorgeschichte ist a priori mit weiteren Darmverlusten zu rechnen.

E Nach einem Volvulus ist es in jedem Falle notwendig, eine Darmwandstufenbiopsie zu entnehmen.

\section{Frage 10}

Im Operationsbericht lesen Sie, dass im Rahmen der Korrektur einer Dünndarmatresie ein Tapering vorgenommen wurde. Was ist gemeint?

A Bei Durchführung der Anastomose führte man zur Kaliberanpassung eine Teilrotation der Darmenden aus.

B Es handelt sich um das antimesenteriale Einschneiden des postatretischen „Mäusedarms“.

C „Tapering“ bezeichnet die Verschmälerung des dilatierten Darmes vor der Anastomose.

D Es handelt sich um eine Y-Anastomose.

E Darunter versteht man allein die Resektion eines massiv überdehnten Darmsegments. 